Please do not remove this page

RMIT

UNIVERSITY

\title{
Study of isothermal vertical bubbly flow using direct quadrature method of moments
}

Deju, Lilunnahar; Cheung, Chi Pok; Yeoh, G.H; Tu, Jiyuan

https://researchrepository.rmit.edu.au/esploro/outputs/9921858932701341/filesAndLinks?institution=61RMIT_INST\&index=null

Deju, L., Cheung, C. P., Yeoh, G. ., \& Tu, J. (2012). Study of isothermal vertical bubbly flow using direct quadrature method of moments. The Journal of Computational Multiphase Flows, 4(1), 23-40.

https://doi.org/10.1260/1757-482X.4.1.23

Document Version: Published Version

Published Version: https://doi.org/10.1260/1757-482X.4.1.23

Repository homepage: https://researchrepository.rmit.edu.au

(c) 2012 Multi-Science Publishing

Downloaded On 2023/04/26 22:05:22 +1000

Please do not remove this page 
Thank you for downloading this document from the RMIT Research Repository.

The RMIT Research Repository is an open access database showcasing the research outputs of RMIT University researchers.

RMIT Research Repository: http://researchbank.rmit.edu.au/

\section{Citation:}

Deju, L, Cheung, S, Yeoh, G and Tu, J 2012, 'Study of isothermal vertical bubbly flow using direct quadrature method of moments', The Journal of Computational Multiphase Flows, vol. 4, no. 1, pp. 23-40.

See this record in the RMIT Research Repository at:

https://researchbank.rmit.edu.au/view/rmit:18014

Version: Published version

\section{Copyright Statement:}

(C) 2012 Multi-Science Publishing

Link to Published Version:

http://dx.doi.org/10.1260/1757-482X.4.1.23 


\title{
Study of Isothermal Vertical Bubbly Flow Using Direct Quadrature Method of Moments
}

\author{
L. Deju', S. C. P Cheung ${ }^{1{ }^{1},}$, G. H. Yeoh ${ }^{2,3}$ and J. Tu ${ }^{1}$ \\ ${ }^{1}$ School of Aerospace, Mechanical and Manufacturing Engineering, \\ RMIT University, VIC 3083, Australia \\ ${ }^{2}$ Australian Nuclear Science and Technology Organization (ANSTO), \\ NSW 2234, Australia \\ ${ }^{3}$ School of Mechanical and Manufacturing Engineering, University of New South Wales, \\ NSW 2052, Australia
}

Received: 12 October 2011, Accepted: 21 February 2012

\begin{abstract}
In the numerical study, investigation of bubbly flow requires deep understanding of complex hydrodynamics under various flow conditions. In order to simulate the bubble behaviour in conjunction with suitable bubble coalescence and bubble breakage kernels, direct quadrature method of moments (DQMOM) has been applied and validated instead. To examine the predictive results from DQMOM model, the validation has been carried out against experimental data of Lucas et al. (2005) and Prasser et al. (2007) measured in the Forschungszentrum Dresden-Rossendorf FZD facility. Numerical results showed good agreement against experimental data for the local and axial void fraction, bubble size distribution and interfacial area concentration profiles. Encouraging results demonstrates the prospect of the DQMOM two-fluid model against flow conditions with wider range of bubble sizes and rigorous bubble interactions. Moreover, moment sensitivity study also has been carried out to carefully assess the performance of the model. In order to perform the moment sensitivity test three different moment criteria has chosen - as 4 moments, 6 moments and 8 moments. Close agreement between the predictions and measurement was found and it appeared that increasing the number of moments does not have much significance to improve the conformity with experimental data. Nonetheless, increasing the number of moments merely contribute to perform the calculation expensive in terms of computational resource and time. Based on the present study, this preliminary assessment has definitely served to demonstrate and exploit DQMOM model's capabilities to handle wider range of bubble sizes as well as moment resolution required to achieve moment independent solution.
\end{abstract}

Keywords: Population balance; bubbly flow; CFD; DQMOM

\section{INTRODUCTION}

Bubble column reactors are known as excellent systems for processes that require large interfacial area for gas- liquid mass transfer and efficient mixing of reacting categories due to a host of gasliquid reactions (oxidations, hydrogenations, halogenations, aerobic fermentations etc.). Multi phase flow is important in bubble column reactors since they often employ to cause high mass and chemical transport rates among liquids and gasses. The transfer rates are a function of the bubble size distribution, mean and fluctuating velocities and trajectories, bubble coalescence and break up, as well as bubble- bubble interactions. For the optimization and rational design of bubble column understanding of bubble mechanistic behaviour represents as a crucial aspect and a greater knowledge on fundamental perceptive of population balance of bubbles is required.

In order to rigorously account for agglomeration and breakage related phenomena the population balance equation (PBE) must be solved along with the continuity and momentum balance equations. Several numerical techniques including the method of moments, the class method, Monte Carlo

*Corresponding Author:

Email address: chipok.cheung@rmit.edu,au 
simulation methods can be found in the literature for solving population balance equations and a lucid description of the mathematical and numerical issues involved can be found in Ramakrishna (2000). The most general formulation is the discretized population balance approach or class method (CM), in which the internal coordinate (e.g., particle length or volume) is discretized into a finite number of size sections (intervals, cells). The application of CM in bubbly flow simulations has been exemplified as MUltiple-SIze-Group (MUSIG) model in several research studies (Olmos, Gentric et al. 2001; Pohorecki, Moniuk et al. 2001; Frank, Shi et al. 2004; Yeoh and Tu 2005; Cheung, Yeoh et al. 2007). The population balance approach based on the MUSIG model has been introduced commercially (Lo 1996) and frequently employed to predict the non-uniform bubble size distribution in a gas-liquid mixture by solving a range of bubble classes. Despite the fact that encouraging results have been reported (Chen, Sanyal et al. 2004; Cheung, Yeoh et al. 2007), in case of flows in large pipe with large bubble diameter, computational resource for solving such large number of transport equations could be extremely excessive. A simple conceptualisation of population balance method namely ABND model has been formulated in handling isothermal gasliquid bubbly flows by Cheung, Yeoh et al. (2007). Rigorous assessment of ABND model has been done by Duan, Cheung et al. (2011), compared with inhomogeneous MUSIG model and experimental data. Encouraging results clearly illustrates the model capability in capturing the dynamical changes of bubble size due to coalescence and breakage. The technique focuses on representing the wide bubble size range with a single average bubble number density quantity, thus the model posed inferior results in terms of Sauter mean bubble diameter. These difficulties associated with method of classes and less accuracy to represent the bubble size range coupled with ABND model leads to the choice of DQMOM model and applied as an alternative to capture the wide bubble size range prevailing in medium and large pipe flows.

One of the most efficient methods developed recently is the direct quadrature method of moments (DQMOM) for solving population balance equation based on a quadrature approximation that provided the best possible closure for the moments of the bubble size distribution for a given number of nodes (Marchisio and Fox 2005). Inspired by the quadrature method of moments (QMOM) (McGraw 1997) DQMOM has been formulated for monovariate and bivariate PBEs. Later the scheme has been extended to solve multivariate PBEs. The DQMOM method condenses the problem substantially by only a small number of moments (i.e. usually four to six lower-order moments) that leads to strong impact on CPU time. Extremely low number of scalars keeps the problem tractable when applied to complicated multiphase flows and in general it does not depend on the lower and upper limit of the classes involved. Thus DQMOM offers a powerful approach for describing polydisperse flow undergoing coalescence and breakage process in the perspective of CFD simulations. The objectives of this paper is to present a comprehensive model validation study to assess the DQMOM model in simulating flow conditions with wider range of bubble sizes and more rigorous bubble interactions. Moreover the work has been carried out to study the effect of number of moments and thus the sensitivity study of bubble size distribution and average mean bubble diameter from various number of moments. Predictions by the DQMOM model validated against gas-liquid flow experiments in vertical pipes of medium size by Lucas, Krepper et al. (2005) and large size of Prasser, Beyer et al. (2007) measured in the Forschungszentrum Dresden-Rossendorf FZD facility.

\section{TWO FLUID MODEL FOR GAS-LIQUID FLOW}

Two sets of conservation equations governing mass and momentum for both gas and liquid continua are needed to be solved for two-fluid model. Denoting the liquid as the continuum phase $\left(\alpha^{1}\right)$ and the gas (i.e. bubbles) as disperse phase $\left(\alpha^{g}\right)$, these equations can be written as:

Continuity equation of liquid phase

$$
\frac{\partial}{\partial t}\left(\rho^{l} \alpha^{l}\right)+\nabla \cdot\left(\rho^{l} \alpha^{l} \mathbf{u}^{l}\right)=0
$$

Continuity equation of gas phase

$$
\frac{\partial}{\partial t}\left(\rho^{g} \alpha^{g}\right)+\nabla \cdot\left(\rho^{g} \alpha^{g} \mathbf{u}^{g}\right)=0
$$


Momentum equation of liquid phase

$$
\frac{\partial}{\partial t}\left(\rho^{l} \alpha^{l} \mathbf{u}^{l}\right)+\nabla \cdot\left(\rho^{l} \alpha^{l} \mathbf{u}^{l} \mathbf{u}^{l}\right)=-\alpha^{l} \nabla P+\alpha^{l} \rho^{l} \mathbf{g}+\nabla \cdot\left[\alpha^{l} \mu_{e}^{l}\left(\nabla \mathbf{u}^{l}+\left(\nabla \mathbf{u}^{l}\right)^{T}\right)\right]+F^{\lg }
$$

Momentum equation of gas phase

$$
\frac{\partial}{\partial t}\left(\rho^{g} \alpha^{g} \mathbf{u}^{g}\right)+\nabla \cdot\left(\rho^{g} \alpha^{g} \mathbf{u}^{g} \mathbf{u}^{g}\right)=-\alpha^{g} \nabla P+\alpha^{g} \rho^{g} \mathbf{g}+\nabla \cdot\left[\alpha^{g} \mu_{e}^{g}\left(\nabla \mathbf{u}^{g}+\left(\nabla \mathbf{u}^{g}\right)^{T}\right)\right]+F^{\mathrm{gl}}
$$

where $\mu_{e}^{l}$ and $\mu_{e}^{g}$ is the effective viscosities of the liquid and gas phases. The total interfacial force $F^{\mathrm{lg}}$ appearing in equation (3) is formulated according to the appropriate consideration of different sub-forces affecting the interface between each phase. For the liquid phase, the total interfacial force is given by:

$$
F^{\mathrm{lg}}=F_{\text {drag }}^{\mathrm{lg}}+F_{\text {lift }}^{\mathrm{lg}}+F_{\text {lubrication }}^{\mathrm{lg}}+F_{\text {dispersion }}^{\mathrm{lg}}
$$

The inter-phase momentum transfer between gas and liquid due to drag force is given by:

$$
F_{\text {drag }}^{\mathrm{lg}}=\frac{1}{8} C_{D} a_{i f} \rho^{l}\left|\mathbf{u}^{g}-\mathbf{u}^{l}\right|\left(\mathbf{u}^{g}-\mathbf{u}^{l}\right)
$$

For vertical gas-liquid flow considered in the present study, the non-drag forces that are the lift, virtual mass, wall lubrication and turbulent dispersion are forces directed perpendicular to the flow direction. Lift force in terms of the slip velocity and the curl of the liquid phase velocity can be described as:

$$
F_{l i f t}^{\lg }=\alpha^{g} \rho^{l} C_{L}\left(\mathbf{u}^{g}-\mathbf{u}^{l}\right) \times\left(\nabla \times \mathbf{u}^{l}\right)
$$

Wall lubrication force, which is in the normal direction away from the heated wall and decays with distance from the wall, is expressed by:

$$
F_{\text {lubrication }}^{\mathrm{lg}}=\frac{\alpha^{g} \rho^{l}\left[\left|\mathbf{u}^{g}-\mathbf{u}^{l}\right|-\left(\left(\mathbf{u}^{g}-\mathbf{u}^{l}\right) \cdot n_{w}\right) n_{w}\right]^{2}}{D_{s}}\left(C_{w 1}+C_{w 2} \frac{D_{s}}{y_{w}}\right) n_{w}
$$

Turbulence induced dispersion based on the consistency of Favre-averaging developed by (Burns, Frank et al. 2004) is applied:

$$
F_{\text {dispersion }}^{\mathrm{lg}}=-C_{T D} C_{D} \frac{\mu_{t}^{g}}{\rho^{g} S c_{b}}\left(\frac{\nabla \alpha^{g}}{\alpha^{g}}-\frac{\nabla \alpha^{l}}{\alpha^{l}}\right)
$$

The drag coefficient $C_{D}$ in equation (6) has been correlated for several distinct Reynolds number regions for individual bubbles according to Ishii and Zuber (1979). The constant $C_{L}$ has been correlated according to Tomiyama (1998) - a relationship expressed as a function of the Eotvos number (Eo) that 
allows positive and negative lift coefficients depending on the bubble size and also accounts for the effects of bubble deformation and asymmetric wake of the bubble. It can be expressed as:

$$
C_{L}= \begin{cases}\min \left[0.288 \tanh \left(0.121 R e_{b}\right), f\left(E o_{d}\right)\right] & E o<4 \\ f\left(E o_{d}\right)=0.00105 E o_{d}^{3}-0.0159 E o_{d}^{2}-0.0204 E o_{d}+0.474 & 4 \leq E o \leq 10 \\ -0.29 & E o>10\end{cases}
$$

where the modified Eotvos number $E o_{d}$ is defined by:

$$
E o_{d}=\frac{g\left(\rho^{l}-\rho^{g}\right) D_{H}^{2}}{\sigma}
$$

in which is the maximum bubble horizontal dimension that can be evaluated through the empirical correlation of Wellek, Agrawal et al. (1966):

$$
D_{H}=D_{s}\left(1+0.163 E o^{0.757}\right)^{1 / 3}
$$

By default, the wall lubrication constants $C_{w 1}$ and $C_{w 2}$ are taken to have values of -0.0064 and 0.016 according to by Krepper, Lucas et al. (2005). The coefficient $C_{T D}$ is normally set to a value of unity and $\mathrm{S}_{\mathrm{cb}}$ is the turbulent bubble Schmidt number with an adopted value of 0.9. Note that for the gas phase, $F_{\mathrm{gl}}=-F_{\mathrm{lg}}$.

In handling bubble induced turbulent flow, unlike single phase fluid flow problem, no standard turbulence model is tailored for gas-liquid flow. Nevertheless, numerical investigation has revealed that standard k- $\varepsilon$ model tends to predict an unrealistically high gas void fraction peak close to the wall (Frank, Shi et al. 2004; Cheung, Yeoh et al. 2007). The Shear Stress Transport (SST) model developed by Menter (1994), which applies the two-equation k- $\omega$ model near the wall and the two-equation k- $\varepsilon$ model in the bulk flow, has been shown to provide more realistic prediction of gas volume fraction or void fraction close to the wall of the flow domain. The SST model is therefore employed herein.

To account for the effect of bubbles on liquid turbulence, the Sato's bubble-induced turbulent viscosity model is employed (Sato, Sadatomi et al. 1981). The turbulent viscosity of liquid phase is thus given by:

$$
\mu_{t}^{l}=\mu_{t s}^{l}+\mu_{t d}^{l}
$$

The shear-induced turbulence is given by:

$$
\mu_{t s}^{l}=C_{\mu} \rho^{l}\left(k^{l}\right)^{2} / \varepsilon^{l}
$$

while the bubble-induced turbulence can be expressed as:

$$
\mu_{t d}^{l}=C_{\mu p} \rho^{l} \alpha^{g} D_{s}\left|\mathbf{u}^{g}-\mathbf{u}^{l}\right|
$$

in which the constants $C_{\mu}$ and $C_{\mu p}$ take on values of 0.09 and 1.2 respectively. For the gas phase, dispersed phase zero equation model is utilized and the turbulent viscosity of gas phase can be obtained as: 


$$
\mu_{t}^{g}=\frac{\rho^{g}}{\rho^{l}} \frac{\mu_{t}^{l}}{\sigma_{g}}
$$

Where $\sigma_{g}$ is the turbulent Prandtl number of the gas phase which has a value of unity.

\section{DIRECT QUADRATURE METHOD OF MOMENTS (DQMOM)}

Population balance equations (PBEs) have found diverse applications in areas involving particulate systems. These equations are particularly useful for situations where particles continually lose their identities, e.g. in crystallizers, liquid-liquid and gas-liquid contactors, microbial fermentors, fluidized beds, polymer reactors (Ramkrishna 1985). In accordance with Fleischer, Becker et al. (1996), the bubble size distribution is calculated with population balance equation that is generally expressed in an integro-differential form describing the local Bubble Size Distribution (BSD) written as

$$
\frac{\partial f(x, \xi, t)}{\partial t}+\nabla \cdot(V(x, \xi, t) f(x, \xi, t))=S(x, \xi, t)
$$

Where $f(x, \xi, t)$ is the bubble number density distribution per unit mixture and bubble volume, $V(x, \xi, t)$ is velocity vector in external space dependent on the external variables $x$ for a given time $t$ and the internal space $\xi$ whose components could be characteristic dimensions such as volume, mass etc. On the right hand side, the term $S(x, \xi, t)$ contains the bubble source/sink rates per unit mixture volume due to the bubble interactions such as coalescence, break-up and phase change.

The direct quadrature method of moments (DQMOM) is based on the direct solution of the transport equations for weights and abscissas of the quadrature approximation (Fan, Marchisio et al. 2004). It shares many features similar to QMOM (McGraw 1997) but in more general in scope for usage in multiphase CFD codes. With the aim to solve multi-dimensional problems, Marchisio and Fox (2005) extended the method where the main idea is to keep track the primitive variables appearing in the quadrature approximation, instead of moments of the BSD. As a result, the evaluation of the abscissas and weights are solved obtained using matrix operations. Since theis method has been described extensively by many authors recently (Marchisio, Pikturna et al. 2003; Fan, Marchisio et al. 2004; Marchisio, Soos et al. 2006). The discussion is limited here to a brief review of the equations. The transport equations for calculating the weights and abscissas are written as (Fan, Marchisio et al. 2004),

$$
\begin{aligned}
& \frac{\partial N_{i}}{\partial t}+\nabla \cdot\left(V_{i}^{d} N_{i}\right)=a_{i} \\
& \frac{\partial \zeta_{i}}{\partial t}+\nabla \cdot\left(V_{i}^{d} \zeta_{i}\right)=b_{i}
\end{aligned}
$$

Where $\zeta_{i}=N_{i} M_{i}$ is the weighted abscissas and the terms $a_{i}$ and $b_{i}$ are related to the birth and death rate of population which forms $2 \mathrm{~N}$ linear equations where the unknowns can be evaluated via matrix inversion to

$$
A \alpha=d
$$

Where the $2 \mathrm{~N}$ vector of unknowns $\alpha$ comprises essentially the terms $a_{i}$ and $b_{i}$ in equations (18) and (19):

$$
\alpha=\left[a_{1} \ldots a_{N} b_{1} \ldots b_{N}\right]^{T}=\left[\begin{array}{l}
a \\
b
\end{array}\right]
$$


In equation (20), the source or sink term is defined by:

$$
d=\left[S_{0} \ldots S_{2 N-1}\right]^{T}
$$

The moment transform of the coalescence and break-up of the term $S_{k}$ can then be expressed as:

$$
S_{k}=\left(B_{k}^{C}-D_{k}^{C}+B_{k}^{B}-D_{k}^{B}\right)
$$

Where the terms $B$ and $D$ represent the birth and death rates of the coalescence and break-up of bubbles is equivalent to $S(x, \xi, t)$ in equation (17). In order to be consistent with the variables used in the two-fluid model, the weights and abscissas can be related to the size fraction of the dispersed phase $\left(f_{k}\right)$ and a variable defined as $\psi_{k}=f_{k} / M_{k}$. As a preliminary study, bubbles are assumed to travel with the gas velocity; the size fraction of $f_{k}$ is related to the weights and abscissas by:

$$
\rho_{g} \alpha_{g} f_{k}=N_{i} M_{i}=\zeta_{k}
$$

On the basis of the approximation given in equation (21), the birth and death rates can be written as:

$$
\begin{gathered}
B_{k}^{C}=\frac{1}{2} \sum_{i} \sum_{j} N_{i} N_{j}\left(M_{i}+M_{j}\right)^{k} a\left(M_{i}, M_{j}\right) \\
D_{k}^{C}=\sum_{i} \sum_{j} M_{i}{ }^{k} a\left(M_{i}, M_{j}\right) N_{i} N_{j} \\
B_{k}^{B}=\sum_{i} \sum_{j} M_{i}^{k} b\left(M_{j}, M_{i}\right) N_{j} \\
D_{k}^{B}=\sum_{i} \sum_{j} M_{i}^{k} b\left(M_{i}, M_{j}\right) N_{i}
\end{gathered}
$$

From above, the weights $N_{i}$ and $N_{j}$ can be determined according to the definition given in equation (21). Coalescence kernel by Prince and Blanch (1990) and break-up mechanism of Luo and Svendsen (1996) were employed to evaluate the above birth and death rates.

\section{MODELLING OF SOURCE TERMS}

\section{a. Bubble Coalescence}

Bubble coalescence occurs via collision of two bubbles which may be caused by wake entrainment, turbulence random collision and buoyancy. Only turbulence random collision is considered in the present study as all bubbles are assumed to be of spherical shape (wake entrainment becomes negligible). The coalescence rate considering the turbulent collision taken from Prince and Blanch (1990) in terms of mass can be expressed as:

$$
a\left(M_{i}, M_{j}\right)=F_{C} \frac{\pi}{4}\left[d_{i}+d_{j}\right]^{2}\left(u_{t i}^{2}+u_{t j}^{2}\right)^{0.5} \exp \left(-\frac{t_{i j}}{\tau_{i j}}\right)
$$


where $\tau_{i j}$ is the contact time for two bubbles given by $\left(d_{i j} / 2\right)^{2 / 3} /\left(\varepsilon^{l}\right)^{1 / 3}$ and $t_{i j}$ is the time required for two bubbles to coalesce having diameters $d_{i}$ and $d_{j}$ estimated to be $\left[\left(d_{i j} / 2\right)^{3{ }^{3}} \rho^{l} / 16 \sigma\right]^{0.5} \ln \left(h_{0} / h_{f}\right)$. The equivalent diameter $d_{i j}$ is calculated as suggested by Chesters and Hofman (1982): $d_{i j}=\left(2 / d_{i}+\right.$ $\left.2 / d_{j}\right)^{-1}$. According to Prince and Blanch (1990), experiments have determined the initial film thickness $h_{\mathrm{o}}=1 \times 10^{-4} \mathrm{~m}$ and critical film thickness $h_{f}=1 \times 10^{-8} \mathrm{~m}$ at which rupture for air-water systems. The turbulent velocity $u_{t}$ in the inertial sub-range of isotropic turbulence which is given by: $u_{t}=\sqrt{2}\left(\varepsilon^{l}\right)^{1 / 3} d^{1 / 3} \cdot F_{C}$ is the coalescence calibration factor.

\section{b. Bubble Breakup}

Bubble break-up rate of volume $v_{j}$ into volume $v_{i}$ is modeled according to Luo and Svendsen (1996), which is based on the assumption of bubble binary break-up under isotropic turbulence situation. The daughter size distribution is accounted using a stochastic break-up volume fraction $f_{B V}$. Denoting the increase coefficient of surface area as $c_{\mathrm{f}}=\left[f_{B V}^{2 / 3}+\left(1-f_{B V}\right)^{2 / 3}-1\right]$, the break-up rate in terms of mass can be obtained as:

$$
r\left(M_{i}, M_{j}\right)=F_{B} C\left(1-\alpha^{g}\right)\left(\frac{\varepsilon}{d_{j}^{2}}\right)^{1 / 3} \int_{\xi_{\min }}^{1} \frac{(1+\xi)^{2}}{\xi^{11 / 3}} \times \exp \left(-\frac{12 c_{f} \sigma}{\beta \rho^{l}\left(\varepsilon^{l}\right)^{2 / 3} d^{5 / 3} \xi^{11 / 3}}\right) d \xi
$$

where $\xi=\lambda / d_{j}$ is the size ratio between an eddy and a particle in the inertial sub-range and consequently $\xi_{\min }=\lambda_{\min } / d_{j}$ and $C$ and $\beta$ are determined from fundamental consideration of drops or bubbles break-up in turbulent dispersion systems to be 0.923 and 2.0. $F_{B}$ is the break-up calibration factor.

Table 1: Inlet boundary conditions of all test cases for numerical simulations.

\begin{tabular}{|c|c|c|c|}
\hline \multicolumn{4}{|c|}{ MTLOOP Experiment } \\
\hline & & Case M107 & $\overline{\text { Case M118 }}$ \\
\hline$\left[\left.\left\langle j_{l}\right\rangle\right|_{Z / D=0}\right]$ & $(\mathrm{m} / \mathrm{s})$ & 1.017 & 1.017 \\
\hline$\left.\left[\left\langle j_{l}\right\rangle\right\rangle_{Z \mid D=0}\right]$ & $(\mathrm{m} / \mathrm{s})$ & 0.140 & 0.219 \\
\hline$\left[\left.\alpha_{g}\right|_{Z / D=0}\right]$ & $(\%)$ & {$[12.1]$} & {$[17.72]$} \\
\hline$\left[\left.D_{s}\right|_{z / D=0.0}\right]$ & $(\mathrm{mm})$ & [5.14] & [6.38] \\
\hline \multicolumn{4}{|c|}{ TOPFLOW Experiment } \\
\hline & & Case T107 & Case T118 \\
\hline$\left.\left[\left\langle j_{l}\right\rangle\right\rangle_{Z \mid D=0}\right]$ & $(\mathrm{m} / \mathrm{s})$ & 1.017 & 1.017 \\
\hline$\left[\left.\left\langle j_{l}\right\rangle\right|_{Z / D=0}\right]$ & $(\mathrm{m} / \mathrm{s})$ & 0.140 & 0.2194 \\
\hline$\left[\left.\alpha_{g}\right|_{Z / D=0}\right]$ & $(\%)$ & {$[12.1]$} & {$[17.72]$} \\
\hline$\left[\left.D_{s}\right|_{z / D=0.0}\right]$ & $(\mathrm{mm})$ & [20.18] & [23.28] \\
\hline
\end{tabular}

\section{OPERATING CONDITIONS AND COMPUTATIONAL DETAILS}

Numerical calculations were achieved through the use of the generic computational fluid dynamics code ANSYS-CFX11. The average bubble number density transport equation with appropriate source and sink terms describing the coalescence and break-up rate of bubble was implemented through the CFX Command Language (CCL). Radial symmetry was assumed in both experimental conditions thereby allowing the computational geometry to be simplified through consideration of 
Table 2: Bubble diameter prediction at different axial location by DQMOM model.

\begin{tabular}{|c|c|c|c|c|}
\hline \multicolumn{5}{|c|}{ MTLOOP Experiment } \\
\hline & & & M107 & M118 \\
\hline \multirow[t]{4}{*}{$Z / D=4.5$} & Experiment & $(\mathrm{mm})$ & {$[5.29]$} & [6.54] \\
\hline & DQMOM (4 MOM) & $(\mathrm{mm})$ & {$[5.68]$} & [7.05] \\
\hline & DQMOM (6 MOM) & $(\mathrm{mm})$ & {$[5.59]$} & [6.60] \\
\hline & DQMOM (8 MOM) & $(\mathrm{mm})$ & {$[5.65]$} & [6.72] \\
\hline \multirow[t]{4}{*}{$Z / D=59.2$} & Experiment & $(\mathrm{mm})$ & {$[5.79]$} & [7.60] \\
\hline & DQMOM (4 MOM) & $(\mathrm{mm})$ & {$[6.43]$} & [7.15] \\
\hline & DQMOM (6 MOM) & $(\mathrm{mm})$ & {$[6.39]$} & [6.39] \\
\hline & DQMOM (8 MOM) & $(\mathrm{mm})$ & {$[6.30]$} & {$[6.86]$} \\
\hline \multicolumn{5}{|c|}{ TOPFLOW Experiment } \\
\hline & & & T107 & T118 \\
\hline \multirow[t]{4}{*}{$Z / D=1.7$} & Experiment & $(\mathrm{mm})$ & {$[19.43]$} & [21.96] \\
\hline & DQMOM (4 MOM) & $(\mathrm{mm})$ & {$[21.54]$} & {$[24.41]$} \\
\hline & DQMOM (6 MOM) & $(\mathrm{mm})$ & {$[22.06]$} & [22.79] \\
\hline & DQMOM (8 MOM) & $(\mathrm{mm})$ & {$[20.96]$} & [23.86] \\
\hline \multirow[t]{4}{*}{$Z / D=39.9$} & Experiment & $(\mathrm{mm})$ & {$[7.49]$} & [7.50] \\
\hline & DQMOM (4 MOM) & $(\mathrm{mm})$ & {$[11.23]$} & [9.57] \\
\hline & DQMOM (6 MOM) & $(\mathrm{mm})$ & {$[10.15]$} & [9.55] \\
\hline & DQMOM (8 MOM) & $(\mathrm{mm})$ & {$[10.21]$} & [9.92] \\
\hline
\end{tabular}

a $60^{\circ}$ radial sector of the pipe with symmetry boundary conditions being imposed at both vertical sides of the computational domain.

Two individual set of experiments that have been performed in the Forschungszentrum DresdenRossendorf FZD facility have been considered for the validation of the simulated results. The experiments are namely - MTLOOP (Lucas, Krepper et al. 2005) and TOPFLOW (Prasser, Beyer et al. 2007). For the MTLOOP experiment, a uniform gas volume fraction was specified at the inlet boundary. On the other hand, 12 equally spaced point sources of the gas phase were placed at the circumference of the $60^{\circ}$ radial sector to represent the wall injection method in TOPFLOW. Gas injection rate at each point source was assumed to be identical. Details of the boundary conditions are summarized in Table 1. Based on grid sensitivity test performed for the MTLOOP and TOPFLOW experiments, grid independent solutions have revealed that computational meshes which consisted of 18,223 elements for MTLOOP and 48,000 elements for TOPFLOW did not appreciably change even though finer computational meshes were tested. For all flow conditions, reliable convergence criterion based on the RMS (root mean square) residual of $1.0 \times 10^{-4}$ was adopted for the termination of numerical calculations. Experimental test cases from MTLOOP are named as Case M107 and Case M118, while test cases from TOPFLOW experiment are represented as Case T107 and Case T118. 
Table 3: Computational time taken by DQMOM model for selected flow conditions.

Computational time for MTLOOP

Number of Moments

Computational time

\begin{tabular}{lc}
\hline DQMOM (4 MOM)-MTLOOP 118 & $3 \mathrm{hrs}$ \\
\hline DQMOM (6 MOM)-MTLOOP 118 & $5 \mathrm{hrs}$ \\
\hline DQMOM (8 MOM)-MTLOOP 118 & $22 \mathrm{hrs}$ \\
\hline
\end{tabular}

Computational time for TOPFLOW

Number of Moments

Computational time

\begin{tabular}{ll}
\hline DQMOM (4 MOM)-TOPFLOW 118 & $14 \mathrm{hrs}$ \\
\hline DQMOM (6 MOM)-TOPFLOW 118 & $42 \mathrm{hrs}$ \\
\hline DQMOM (8 MOM)-TOPFLOW 118 & $68 \mathrm{hrs}$
\end{tabular}

(a)

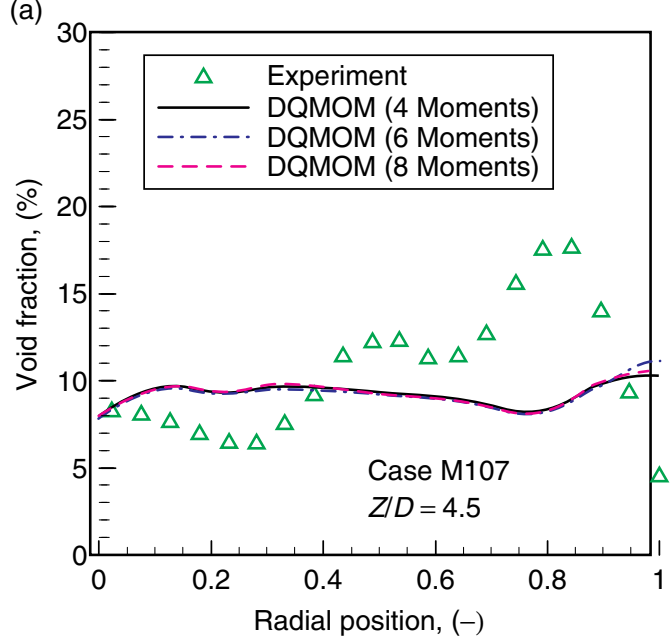

(c)

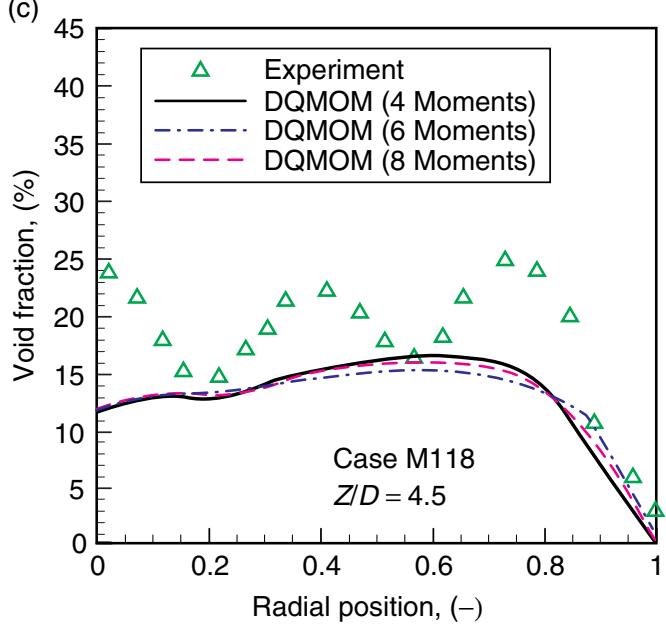

(b)

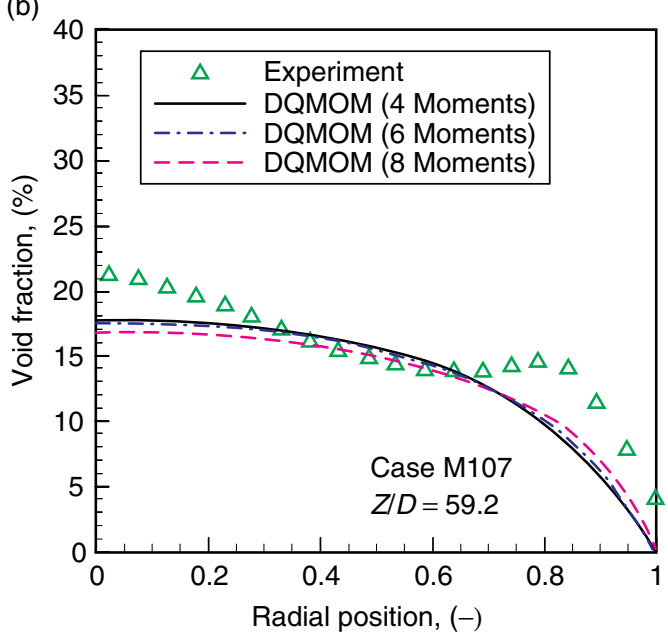

(d)

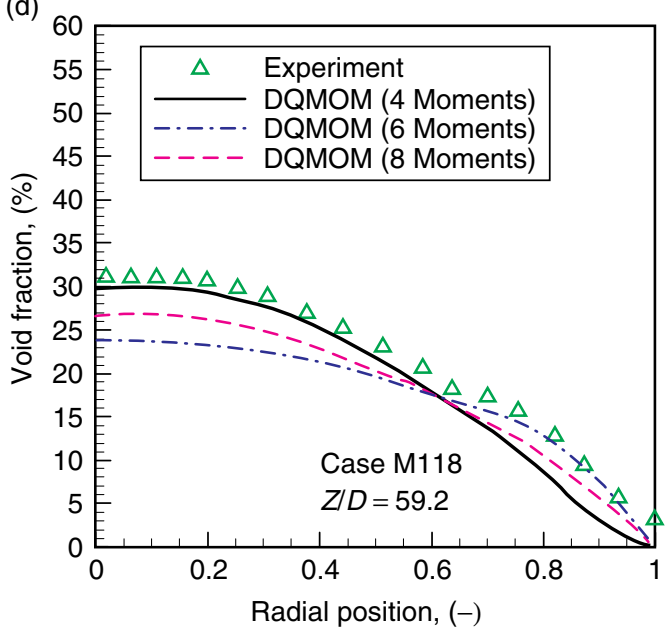

Figure 1: Comparison of the predicted radial gas volume fraction distributions of the DQMOM model with the MTLOOP measurments. 


\section{RESULTS AND DISCUSSION}

\section{a. Test case I: MTLOOP Experiment}

The predicted results obtained with the DQMOM model are compared with the measurements available in the literature (Lucas, Krepper et al. 2005; Duan, Cheung et al. 2011) for both qualitative and quantitave comparisons. The geometry is as used by Lucas, Krepper et al. (2005), a vertical medium size cylindrical pipe with a height of $3500 \mathrm{~mm}$ and an inner diameter of 51.2 $\mathrm{mm}$. The schematic diagram of the MTLOOP experiment can be found out from Lucas, Krepper et al. (2005). 19 capillaries with an inner diameter of $0.8 \mathrm{~mm}$ were equally distributed over the cross section area of the pipe and air was injected through these capillaries via an injection device. An electrode wire mesh sensor was placed above a certain distance from the injection device that helped to measure the local instantaneous gas fraction, void fraction and as well as bubble size distribution. As in the experiment, the air-water system has been chosen and the superficial gas and liquid velocity are varied from $14 \mathrm{~m} / \mathrm{s}$ to $4 \mathrm{~m} / \mathrm{s}$ respectively.

In general, it has been observed that DQMOM model were capable to capture the transition process from 'wall peak' to 'core peak' of the gas volume fraction distribution (Serizawa and Kataoka 1988). While approaching the wall (Figure 1), as the breakage phenomena dominates over coalescence, the bubble size decreases and thus creating the radial bubble seggragation. In

(a)

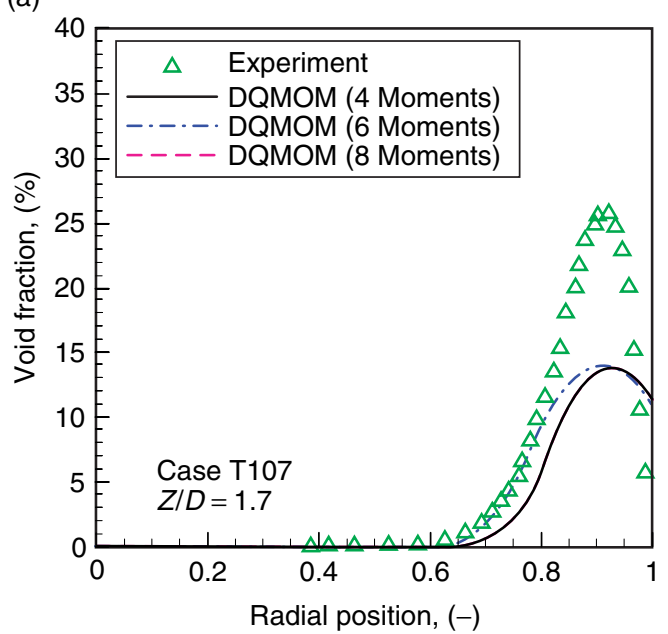

(c)

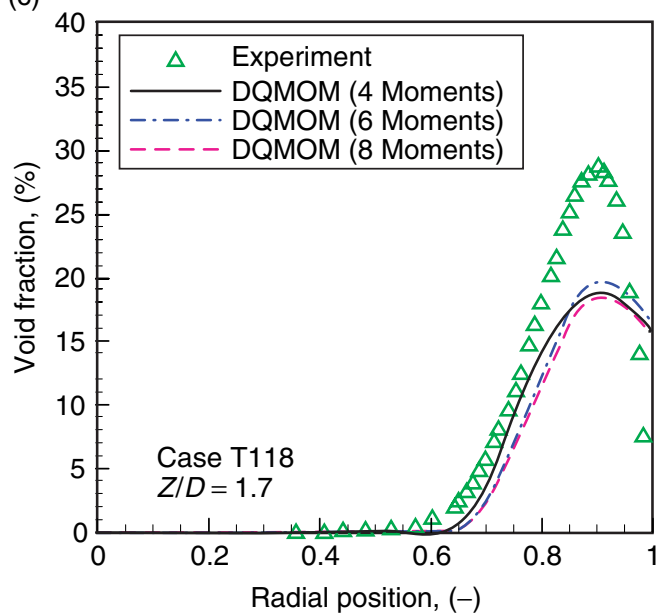

(b)

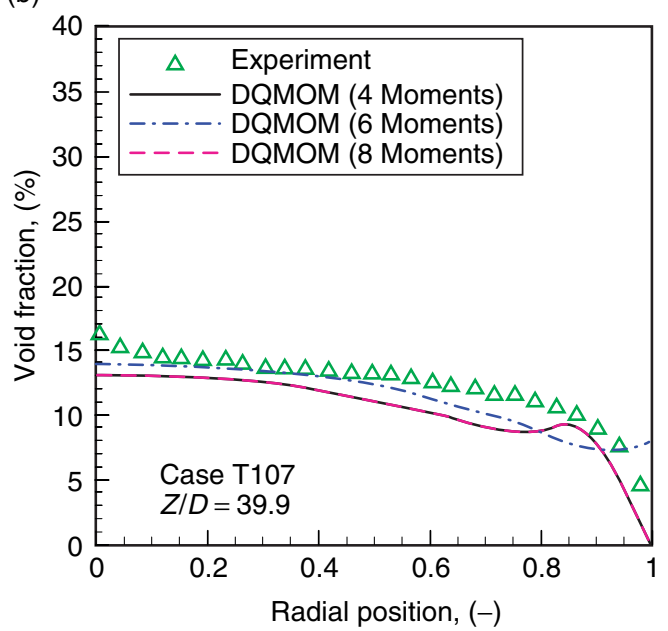

(d)

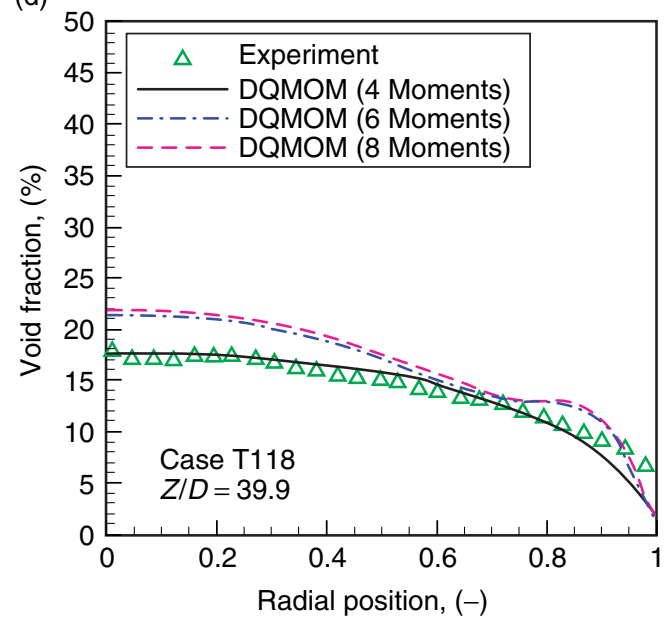

Figure 2: Comparison of the predicted radial gas volume fraction distributions of the DQMOM model with the TOPFLOW measurments. 
the contrary, while approaching to the center of the bubble column, coalescence dominates due to high volume fraction of gas and therefore bubble size increases. As illustrated in Figure 5, predicted gas volume fracion profile through axial direction is also in good agreement with measurement.

Table 2 gives the value of bubble diameter prediction with the corresponding moments. In the present study and for the given geometry the number of moments was limited to 8 as shown in the table. By comparison with the experimental data with the DQMOM method, it does not apprear that increasing the number of moments further increases significantly the agreement and seems to be within acceptable range. Additionally, as can be seen from Table 3, increasing the number of moments to 8 the calculation becomes computationally expensive when compared to the much faster DQMOM method with 4 or 6 moments.

As shown in Figure 3, the bubble size distribution has significantly widened after a series of merging procedure. Especially for case M118, the bubble size range has achieved almost twice of its initial size range. The clearly demonstrate that the evolution of bubble size distribution was dominated by bubble coalescence. At different stipulated flow conditions, the predictive capability for DQMOM method for 4, 6 and 8 moments has been demonstrated and it revealed that the results were comparable reasonably well with the experimental data. It has been found that 4 and 6 moments

(a)

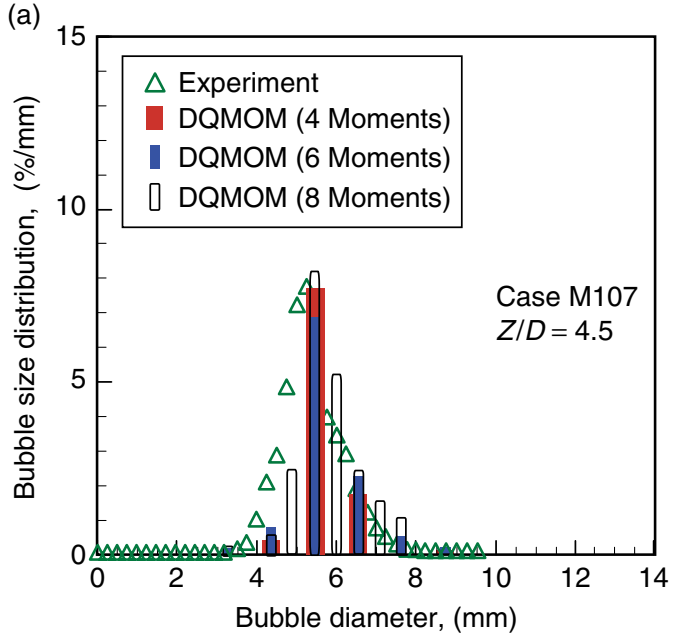

(c)

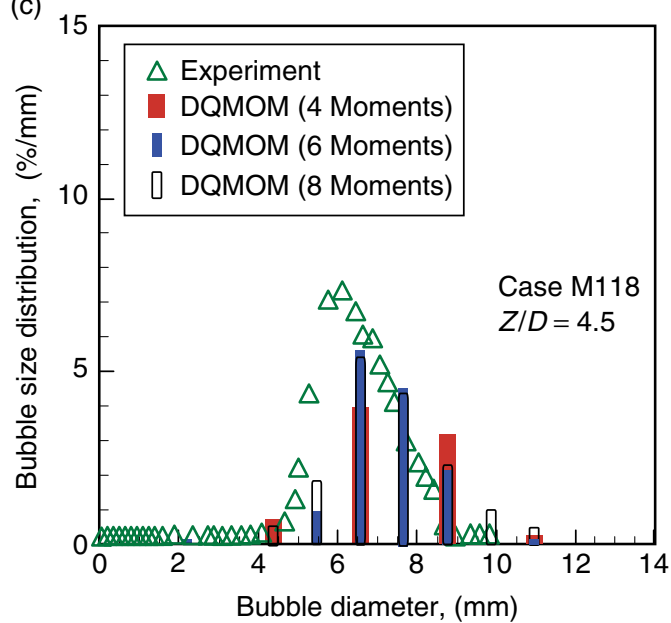

(b)

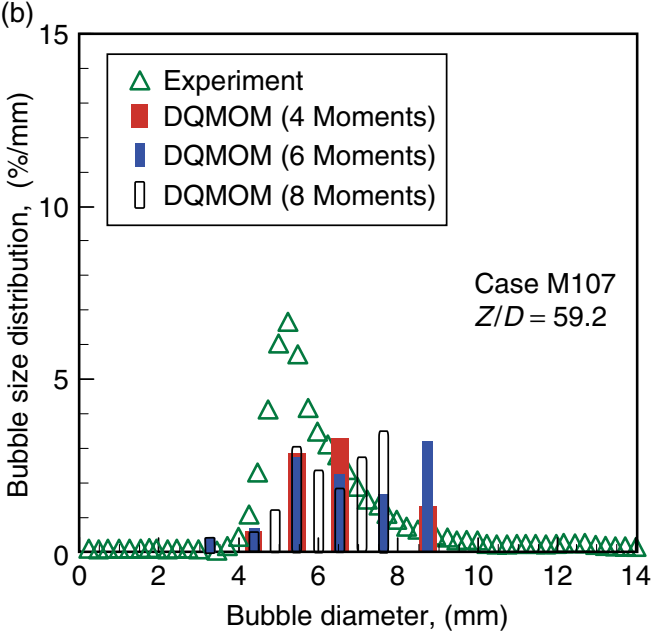

(d)

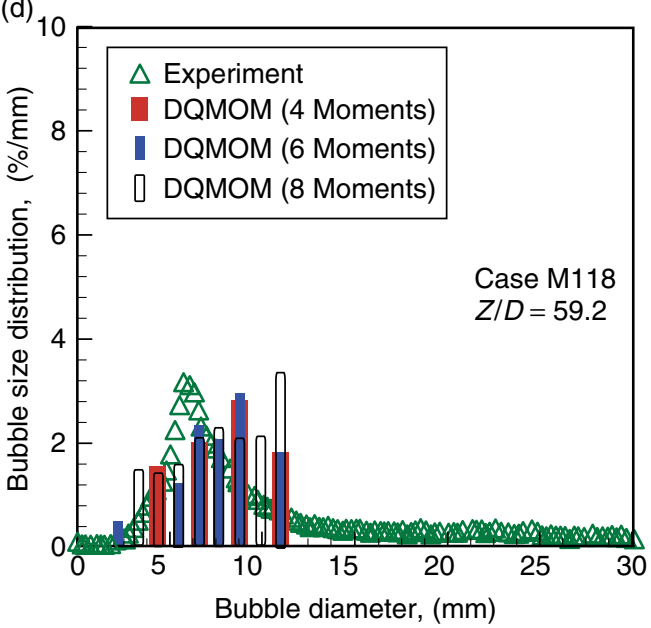

Figure 3: Comparison of predicted bubble size distribution of the DQMOM model with the MTLOOP experiment data. 
give quite better results throughout the comparisons with experimental data under the conditions studied in the present work. Using more than 6 moments (i,e 8 moments) gives also good results but the computational efforts gradually becomes excessive.

\section{b. Test case II: TOPFLOW Experiment}

In order to validate the DQMOM model predicted results with wider range of bubble sizes, the experimental setup of Prasser, Beyer et al. (2007) has been used and the measurement results can be find out from Prasser, Beyer et al (2007) and Duan, Cheung et al. (2011). The experimental setup consists of a large size vertical cylindrical pipe with height $9000 \mathrm{~mm}$ and inner diameter of $195.3 \mathrm{~mm}$. The maximum superficail velovitied for gas and liquid phase were indentical to the MTLOOP experiment and the water circulation (from bottom to the top at constant temperature of $30^{\circ} \mathrm{C}$ ) was maintained by a heat exchanger installed in the water reservoir. However a different gas injection device was employed with variable injection system that was constructed by equipping with gas injection units at 18 different axial positions from $Z / D=1.1$ to 9.9. Each injection unit was consist of three levels of air chambers. The upper and the lower chambers have 72 annular distributed orifices of $1 \mathrm{~mm}$ diameter for small bubble injection while the central chamber has 32 annularly distributed orifices of $4 \mathrm{~mm}$ diameter for large bubble injection. Instantaneous information of gas volume fraction as well as bubble size distribution was gathered by using a fixed wire-mesh sensor implemented at the top of the pipe.
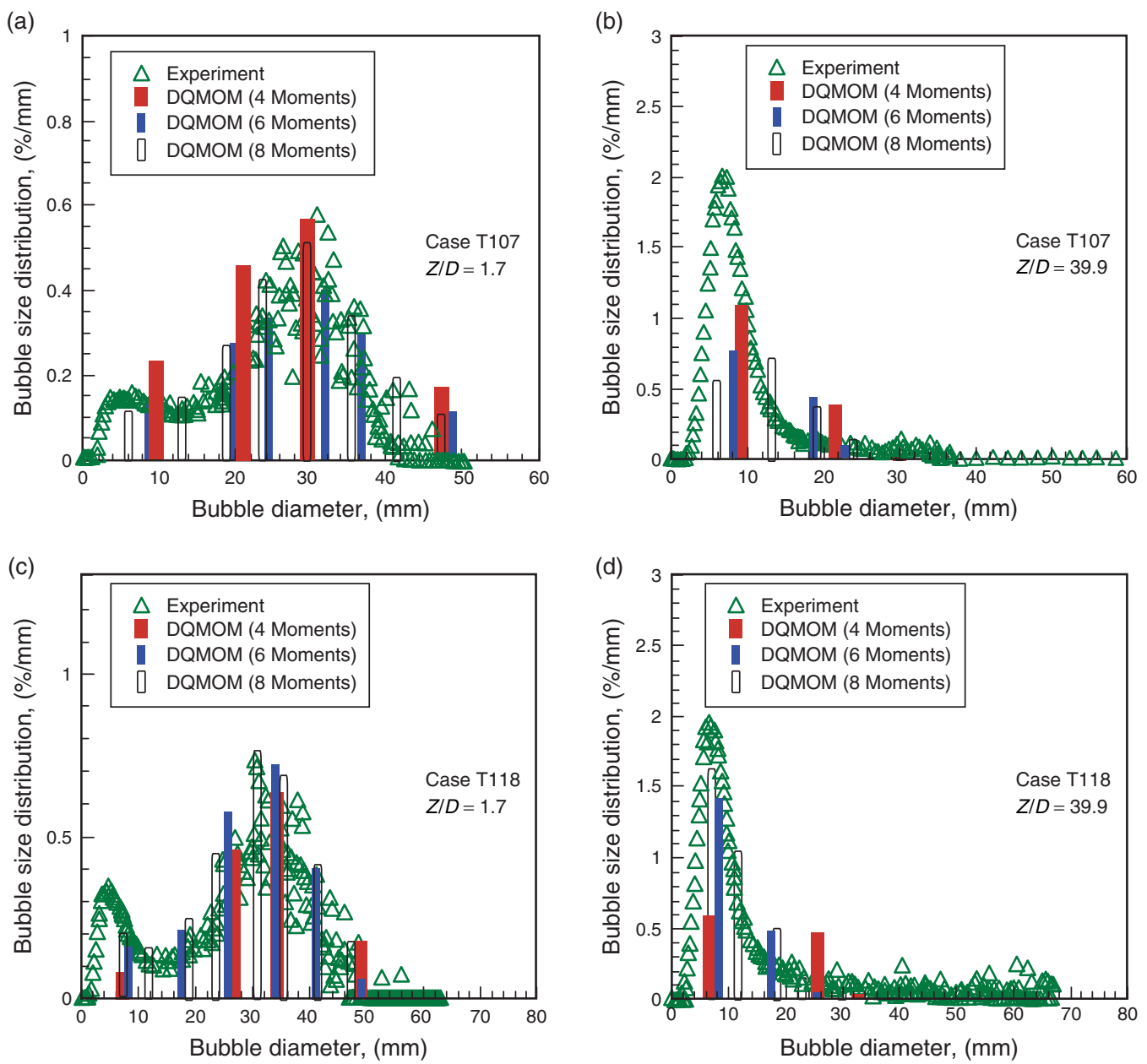

Figure 4: Comparison of predicted bubble size distribution of the DQMOM model with TOPFLOW experiment data. 
(a)

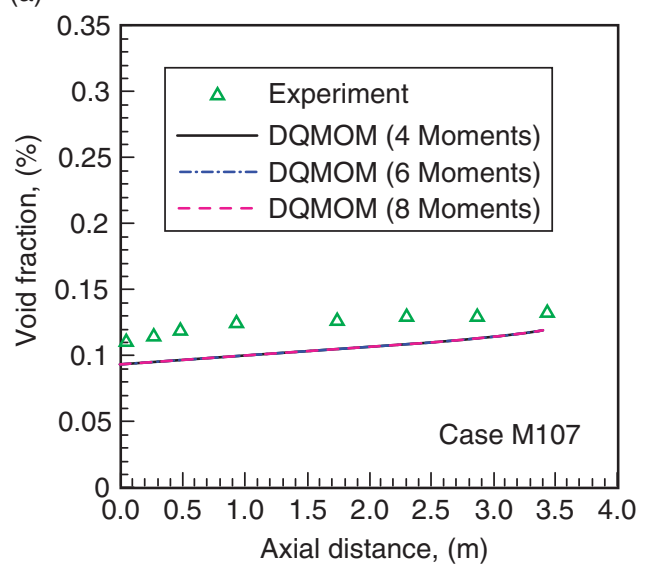

(c)

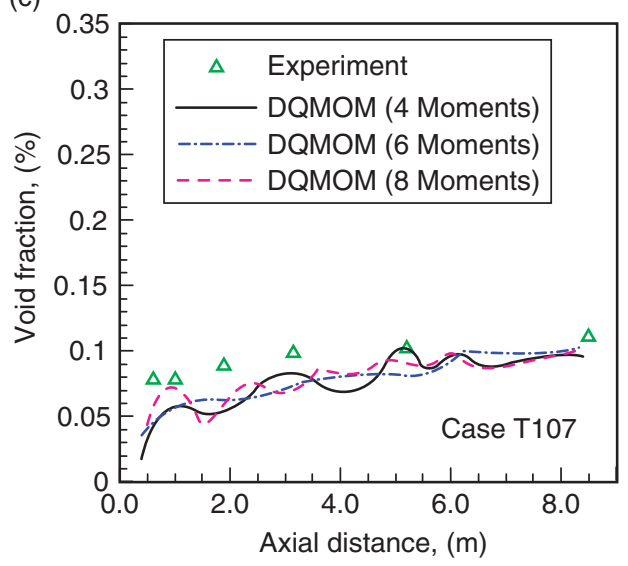

(b)

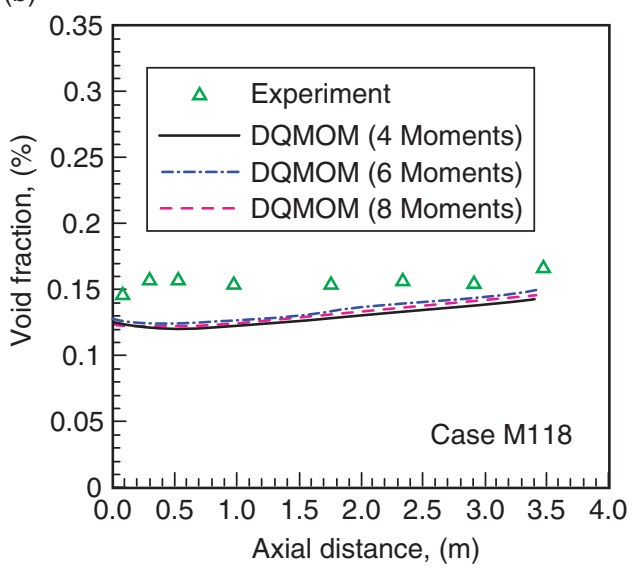

(d)

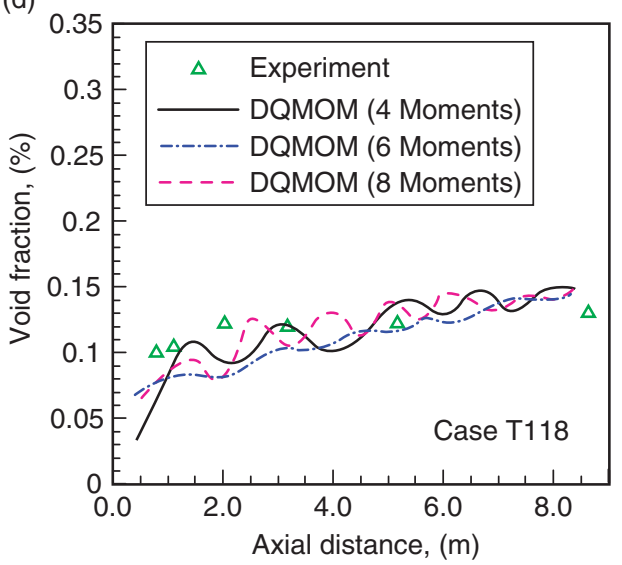

Figure 5: Comparison of the predicted cross-sectional averaged gas volume fraction profiles with both MTLOOP and TOPFLOW measurements along axial direction.

A void fraction distribution comparison between DQMOM and experimental data based on TOPFLOW experiment is presented in Figure 2. Due to the fact that bubbles were injected from the gas injection orifices located on the circumference of the pipe, highly concentrated bubbles were formed near the wall and resulted in 'wall peak' void fraction distribution close to the inlet of the pipe. In general, prediction from the DQMOM model was reasonably well agreed with the measurement for different number of moments (4-8 moments).

As stated earlier, the gas injection orifices located on the circumference, closed packed swarm of bubbles was formed and then immediately merged with others forming larger bubbles. This rapid coalescence of bubbles exposes a bimodal bubble size disribution with a wide spectrum of bubble size range. As demonstraed in Figure 4, the bimodal distributed bubbles from the injection unit gradually collapsed to single-peaked distribution near the outlet of the pipe. Thus bubble break-up was ascertained to be the dominant mechanism within the gas-liquid flow for TOPFLOW experiment.

The local interfacial area concentration can be calculated from lcoal void fraction and Sauter mean bubble diameter according to $a_{i f}=6 \alpha_{g} / D_{s}$ (Assuming that all bubbles are spherical). Figure 6 illustrates the measured and predicted local IAC profles for the respective two axial locations. The IAC profile approximately went along with the same trend as void fracion profile. In high void fraction cases, large distorted bubbles start to emerge introducing errors in IAC prediction. That may resulted the slight difference between predicted and measured data. 
(a)

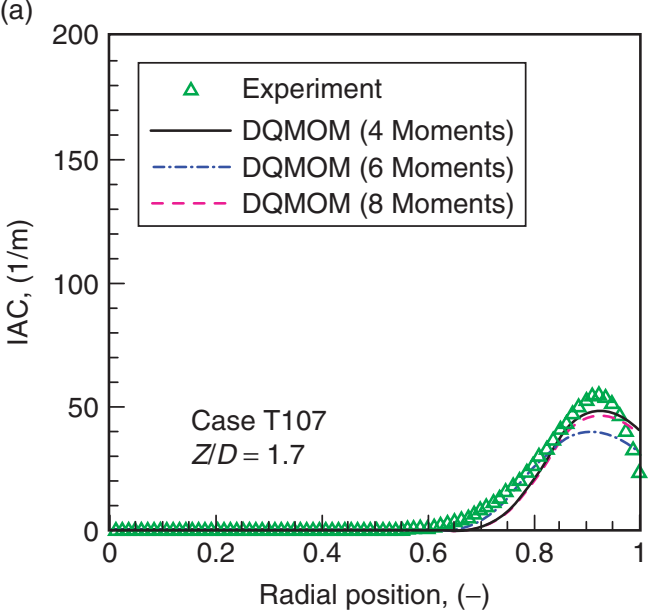

(c)

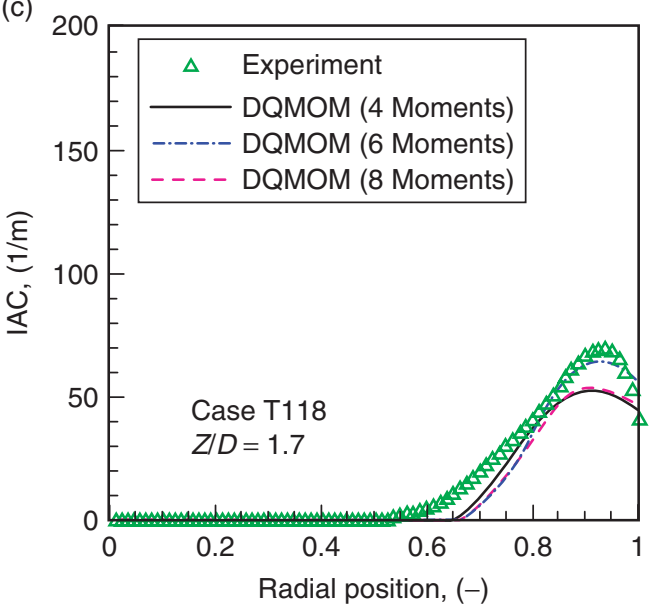

(b)

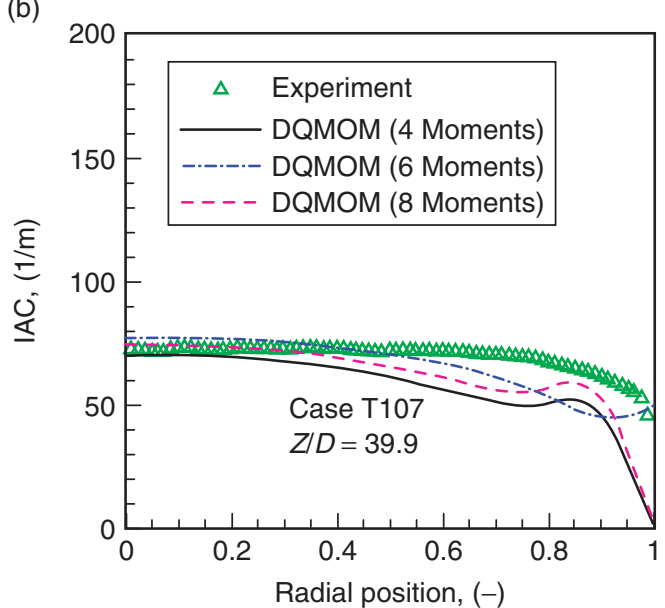

(d)

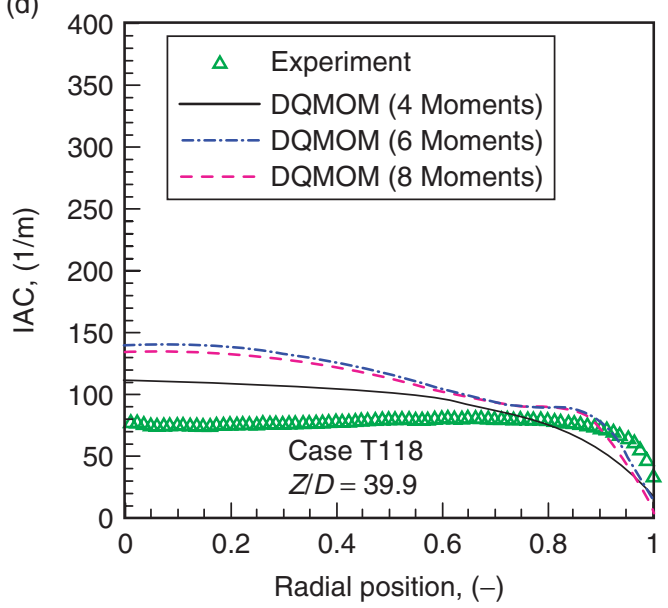

Figure 6: Comparison of the predicted radial interfacial area concentration profile with TOPFLOW measurements.

\section{CONCLUSION}

A comparison between the measured and predicted result for the local radial distributions of void fraction, IAC and bubble size distribution from DQMOM model has been presented. The performance of the DQMOM model has also been tested with four, six and eight moments. Considering the increase in computational time with an increasing number of moments, six moments appears to be a decent choice for moment independent result.

From the above numerical study, some limitation of the current coalescence and breakage kernels has been revealed. For bubbly simulations presented in this paper, coalescence and breakage kernel were derived based on the spherical bubble assumption that limits the kernels to be applicable to bubbly flow regime where bubbles are subject to the balance of the surface tension and surrounding fluid motion. Thus, coalescence due to wake entrainment may become significant which unfortunately was not modelled in current adopted kernels.

Moreover the current models were also exposed to the limitation of interfacial force model developed and calibrated from isolated single particle which may not be strictly applicable if particles are closely packed. According to (Simonnet, Gentric et al. 2007), when gas volume fraction exceeded the critical value of around 15\% - the drag force acting on bubbles decreased significantly. Without considering the influence of neighbouring bubbles, these models may introduce errors to the interfacial momentum transfer calculation.

In summary, the DQMOM model coupled with the bubble coalescence and breakup kernels and the interphase force formulations presented in this paper has the ability to reasonably predict the hydrodynamics of complex gas-liquid flows. Thus DQMOM approach stands out as a unified description to represent itself as a rather sound mathematical approach to handle rigorous bubble 
interaction and an elegant tool to solve the population balance equation with relatively less computational burden. Further work will focus on improving the models for bubble coalescence and breakup and interfacial forces on bubble swarms in order to yield more realistic prediction.

\section{ACKNOWLEDGEMENT}

The financial support provided by the Australian Research Council (ARC project ID DP0877734) is gratefully acknowledged. The experiments and data evaluation were carried out in the frame of a research project funded by the German Federal Ministry of Economics and Technology, project number 1501329.

\section{NOMENCLATURE}

$\begin{array}{ll}a & \text { birth rate } \\ \mathrm{b} & \text { breakage rate } \\ a_{i f} & \text { interfacial area concentration } \\ \mathrm{B}^{\mathrm{B}} & \text { Mass production rate due to breakage } \\ \mathrm{B}^{\mathrm{C}} & \text { Mass production rate due to coalescence } \\ C_{D} & \text { drag coefficient } \\ C_{L} & \text { Lift coefficient } \\ C_{W 1}, C_{W 2} & \text { Wall lubrication Constant } \\ D^{B} & \text { Mass loss rate due to breakage } \\ D^{C} & \text { Mass loss rate due to coalescence } \\ D_{H} & \text { Maximum bubble horizontal dimension } \\ D_{s} & \text { Bubble Sauter mean diameter } \\ E_{o} & \text { Eötvos number } \\ E_{o d} & \text { Modified Eötvos number } \\ f & \text { Bubble size distribution } \\ F & \text { Total interfacial force } \\ F_{\mathrm{lg}}^{\mathrm{drag}} & \text { Drag force } \\ F_{\mathrm{lg}}^{\mathrm{lift}} & \text { Lift force } \\ F_{\mathrm{lg}}^{\mathrm{lu}} & \text { Wall lubrication force } \\ F_{\mathrm{lg}}^{\mathrm{di} p e r s i o n} & \text { Turbulent dispersion force } \\ g & \text { Gravitational acceleration } \\ \mathrm{k} & \text { turbulent kinetic energy } \\ \mathrm{M} & \text { mass scale of gas phase (bubble) } \\ n, N & \text { Average number density of gas phase } \\ \mathrm{n}_{\mathrm{w}} & \text { Outward vector normal to the wall surface } \\ \mathrm{P} & \text { Pressure } \\ R & \text { Sink and source term } \\ R e_{b} & \text { Local Reynolds number } \\ \mathrm{S} & \text { Mass transfer rate due to coalescence and breakage } \\ t & \text { Bubble contact time } \\ \mathrm{u} & \text { Velocity } \\ \mathrm{y}_{\mathrm{w}} & \text { Adjacent point normal to wall surface } \\ & \end{array}$

Greek Symbols

$\begin{array}{ll}\alpha & \text { Void fraction } \\ \varepsilon & \text { Turbulence kinetic energy dissipation } \\ \rho & \text { Density } \\ \sigma & \text { Surface tension } \\ \zeta & \text { Weighted abscissa } \\ \xi & \text { Size fraction between an eddy and a particle } \\ \text { Subscripts } & \\ \mathrm{g} & \text { Gas } \\ \mathrm{gl} & \text { Transfer of quantities from liquid phase to vapour phase }\end{array}$


i $\quad$ Bubble size class

j $\quad$ Velocity group

$1 \quad$ Liquid

$\lg \quad$ Transfer of quantities from gas phase to liquid phase

\section{REFERENCES}

Burns, A. D., T. Frank, et al. (2004). The Farve averaged drag model for turbulent dispersion in Eulerian Mulit-phase flows. Proceeding of the Fifth International Conference on Multiphase flow.

Chen, P., J. Sanyal, et al. (2004). "CFD modeling of bubble columns flows: implementation of population balance." Chemical Engineering Science 59: 5201-5207.

Chesters, A. K. and G. Hofman (1982). "Bubble Coalescence in Pure Liquids." Applied Scientific Research 38: 353-361.

Cheung, S. C. P., G. H. Yeoh, et al. (2007). "On the modelling of population balance in isothermal vertical bubbly flows Average bubble number density approach.” Chemical Engineering and Processing 46(8): 742-756.

Cheung, S. C. P., G. H. Yeoh, et al. (2007). "On the numerical study of isothermal vertical bubbly flow using two population balance approaches." Chemical Engineering Science 62(17): 4659-4674.

Duan, X. Y., S. C. P. Cheung, et al. (2011). "Gas-liquid flows in medium and large vertical pipes." Chemical Engineering Science 66(5): 872-883.

Fan, R., D. L. Marchisio, et al. (2004). "Application of the direct quadrature method of moments to polydisperse gas-solid fluidized beds." Powder Technology 139(1): 7-20.

Fleischer, C., S. Becker, et al. (1996). "Detailed modeling of the chemisorption of $\mathrm{CO} 2$ into $\mathrm{NaOH}$ in a bubble column." Chemical Engineering Science 51(10): 1715-1724.

Frank, T., J. Shi, et al. (2004). Validation of Eulerian multiphase flow models for nuclear safety application. Proceeding of International Symposium on Two-Phase Modelling and Experimentation.

Ishii, M. and N. Zuber (1979). "Drag coefficient and relative velocity in bubbly, droplet or particulate flows." Aiche Journal 25(5): 843-855.

Krepper, E., D. Lucas, et al. (2005). "On the modelling of bubbly flow in vertical pipes." Nuclear Engineering and Design 235(5): 597-611.

Lo, S. M. (1996). "Application of Population Balance to CFD Modelling of Bubbly Flow via the MUSIG model." AEA Technology, AEAT-1096.

Lucas, D., E. Krepper, et al. (2005). "Development of co-current air-water flow in a vertical pipe.” International Journal of Multiphase Flow 31(12): 1304-1328.

Luo, H. and H. F. Svendsen (1996). "Theoretical model for drop and bubble breakup in turbulent dispersions." Aiche Journal 42(5): 1225-1233.

Marchisio, D. L. and R. O. Fox (2005). "Solution of population balance equations using the direct quadrature method of moments." Journal of Aerosol Science 36(1): 43-73.

Marchisio, D. L., J. T. Pikturna, et al. (2003). "Quadrature method of moments for population-balance equations." Aiche Journal 49(5): 1266-1276.

Marchisio, D. L., M. Soos, et al. (2006). "Role of turbulent shear rate distribution in aggregation and breakage processes." Aiche Journal 52(1): 158-173.

McGraw, R. (1997). "Description of aerosol dynamics by the quadrature method of moments." Aerosol Science and Technology 27(2): 255-265.

Menter, F. R. (1994). "2-Equation eddy-visocity turbulence models for engineering applications.” Aiaa Journal 32(8): $1598-1605$.

Olmos, E., C. Gentric, et al. (2001). "Numerical simulation of multiphase flow in bubble column reactors. Influence of bubble coalescence and break-up." Chemical Engineering Science 56(21-22): 6359-6365.

Pohorecki, R., W. Moniuk, et al. (2001). "Modelling of the coalescence/redispersion processes in bubble columns." Chemical Engineering Science 56(21-22): 6157-6164.

Prasser, H. M., M. Beyer, et al. (2007). "Evolution of the structure of a gas-liquid two-phase flow in a large vertical pipe." Nuclear Engineering and Design 237(15-17): 1848-1861.

Prince, M. J. and H. W. Blanch (1990). "Bubble Coalescence and Break-up in Air-Sparged Bubble-Columns.” Aiche Journal 36(10): 1485-1499.

Ramakrishna, D. (2000). Population Balances, Academic Press, London. 
Ramkrishna, D. (1985). “The status of population balances.” Rev. Chem. Engng 3: 49-95.

Sato, Y., M. Sadatomi, et al. (1981). "Momentum and Heat-Transfer in 2-Phase Bubble Flow .1. Theory." International Journal of Multiphase Flow 7(2): 167-177.

Serizawa, A. and I. Kataoka, Eds. (1988). Phase distribution in two-phase flow. Transient Phenomena in Multiphase Flow. New York, Hemisphere Pub. Corp.

Simonnet, M., C. Gentric, et al. (2007). "Experimental determination of the drag coefficient in a swarm of bubbles." Chemical Engineering Science 62(3): 858-866.

Tomiyama, A. (1998). Struggle with computational bubble dynamics. Third International Conference on Multiphase Flow. Lyon, France 157.

Wellek, R. M., A. K. Agrawal, et al. (1966). "Shape of liquid drops moving in liquid media.” Aiche Journal 12(5): 854-\&.

Yeoh, G. H. and J. Y. Tu (2005). "Thermal-hydrodynamic modeling of bubbly flows with heat and mass transfer." Aiche Journal 51(1): 8-27. 